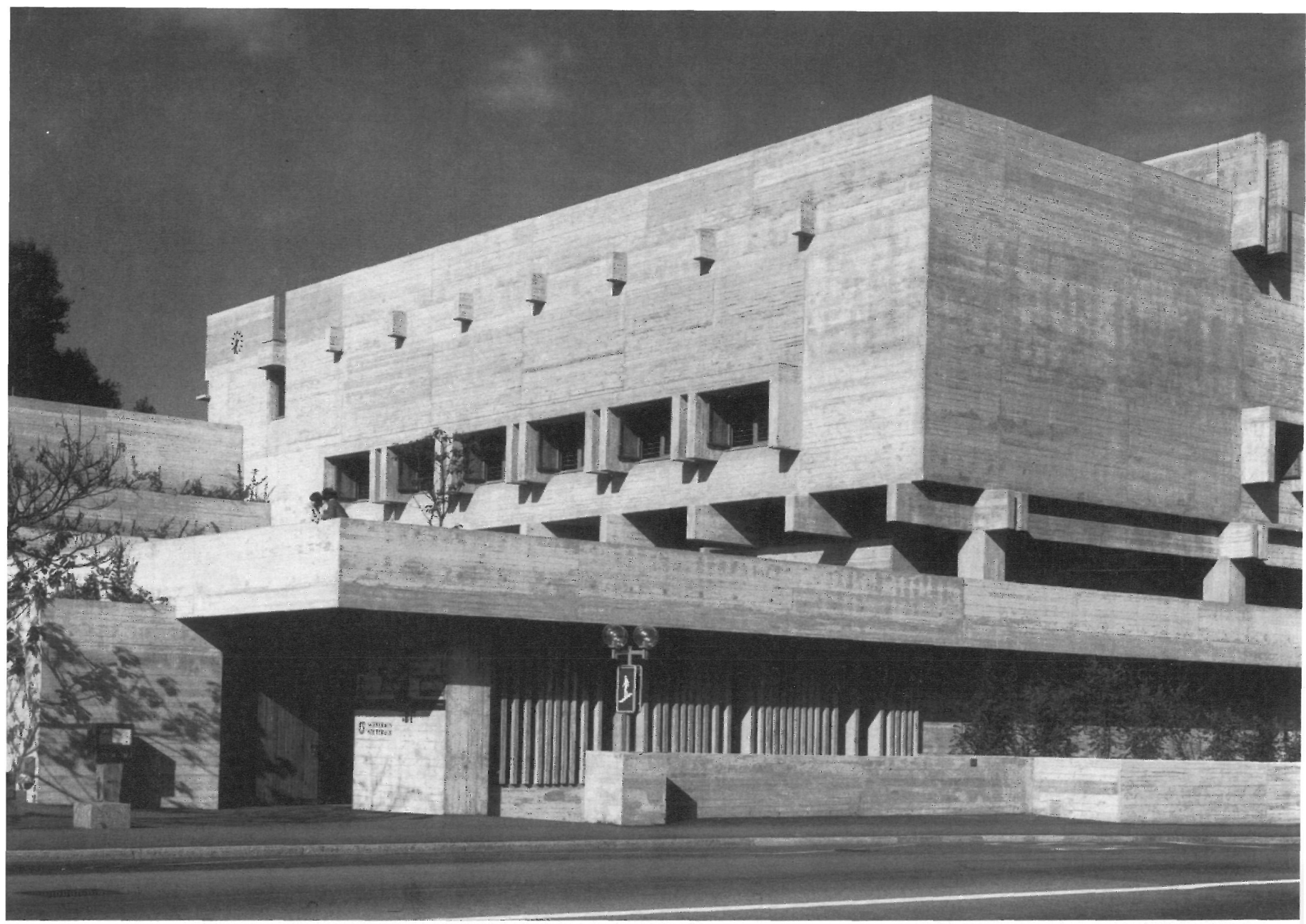

\title{
escuela secundaria Stettbach
}

Zurich * Suiza

RUDOLF y ESTHER GUYER, arquitectos

$142-122$

Sinopsis Este complejo escolar se compone de tres núcleos claramente diferenciados e independientes, separados entre sí por espacios verdes, públicos o privados. Las construcciones ocupan el $10 \%$ del solar, quedando el resto para jardines y campos de juego.

El núcleo principal del conjunto es la escuela secundaria, con capacidad para 720 alumnos, 24 clases y diversos salones destinados a funciones complementarias, tales como: labores femeninas, dibujo, enseñanzas prácticas, carpintería, almacenes,

El gimnasio consta de tres salas para ejercicios gimnásticos, convertibles en una sola para deportes de competición, una estación sanitaria de primeros auxilios, una biblioteca, talleres, aparcamiento para bicicletas y servicios anexos.

El núcleo destinado a jardín de infancia se compone de equipos de juego, vestuarios y aseos.

La edificación se destaca exteriormente por el equilibrio logrado entre las construcciones acabadas en hormigón visto, y los amplios espacios verdes y patios al aire libre. En el interior se advierte un aprovechamiento máximo de la riqueza plástica expuestos, dando lugar a interesantes planteamientos formales.

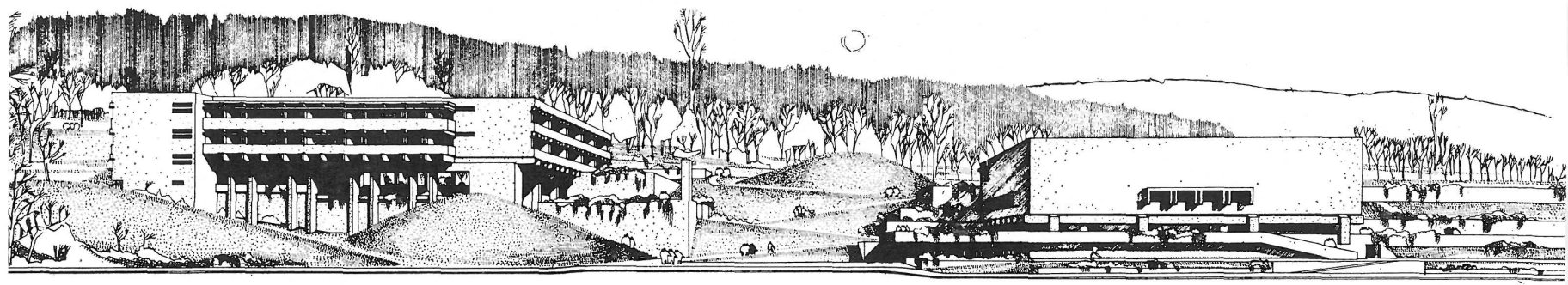




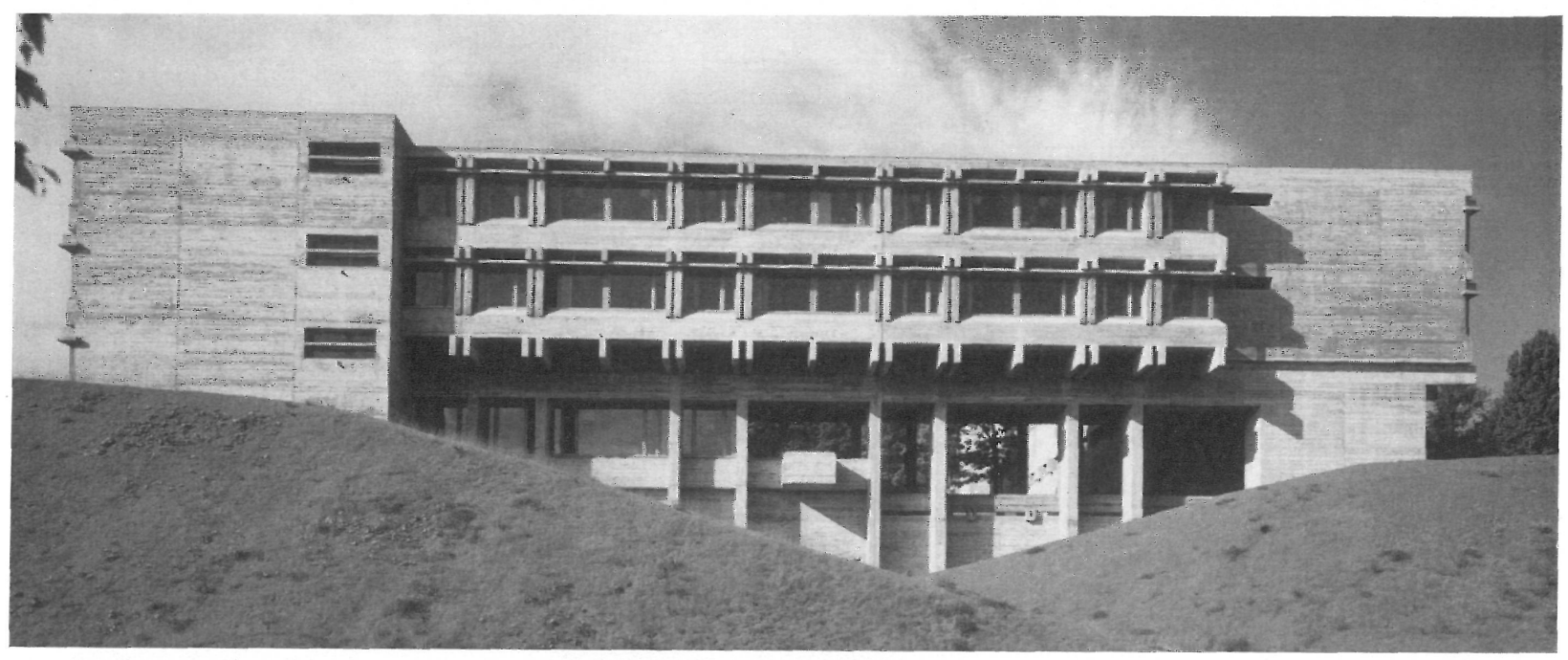

Este complejo escolar, realizado en fases superpuestas, con una duración total de las obras de aproximadamente tres años, se halla emplazado en una parcela de $26.600 \mathrm{~m}^{2}$ de superficie, de los cuales 2.450 fueron ocupados por la edificación, quedando el resto, que sobrepasa el $90 \%$ del total, destinado a jardines, paseos, zonas deportivas y espacios verdes públicos.

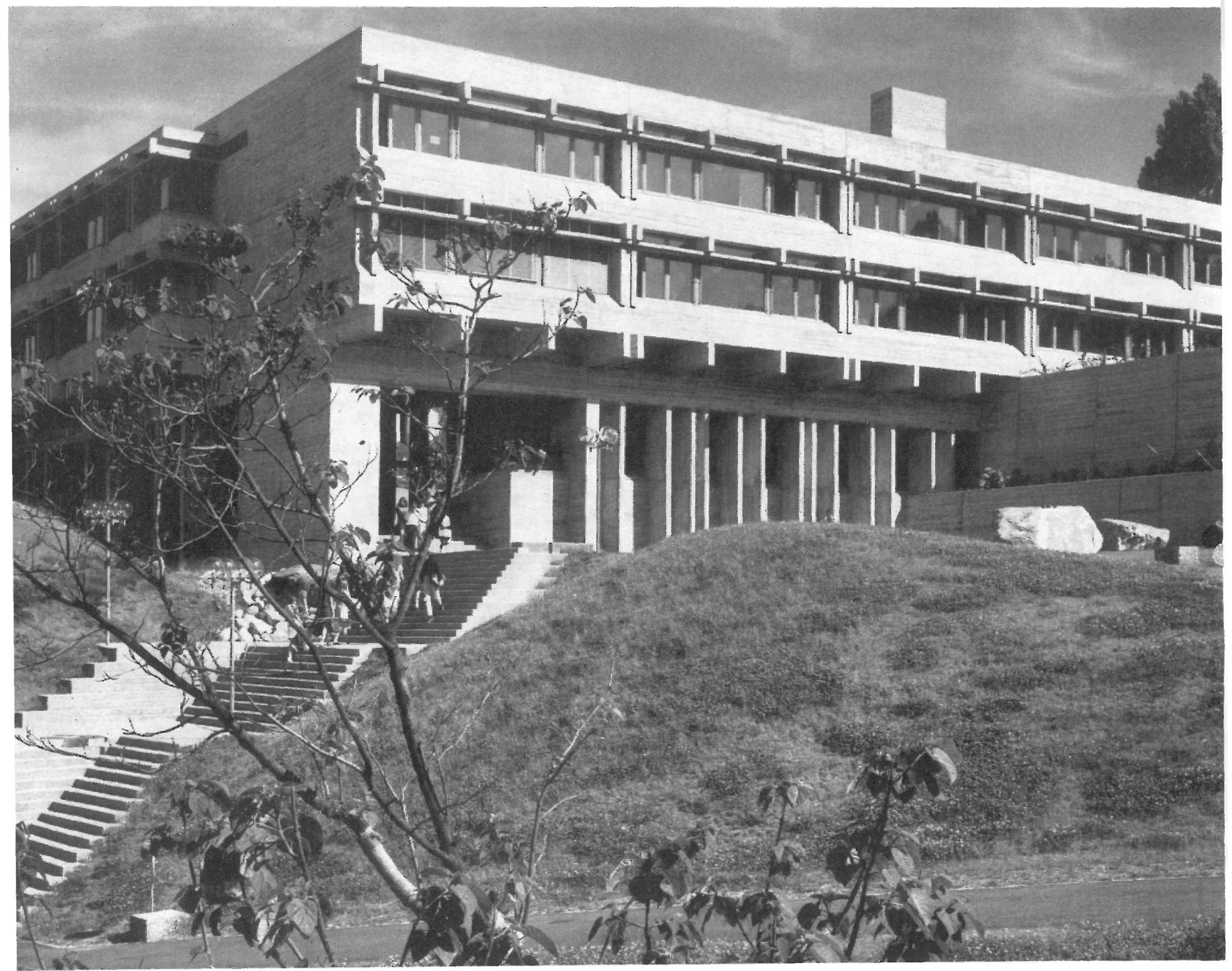



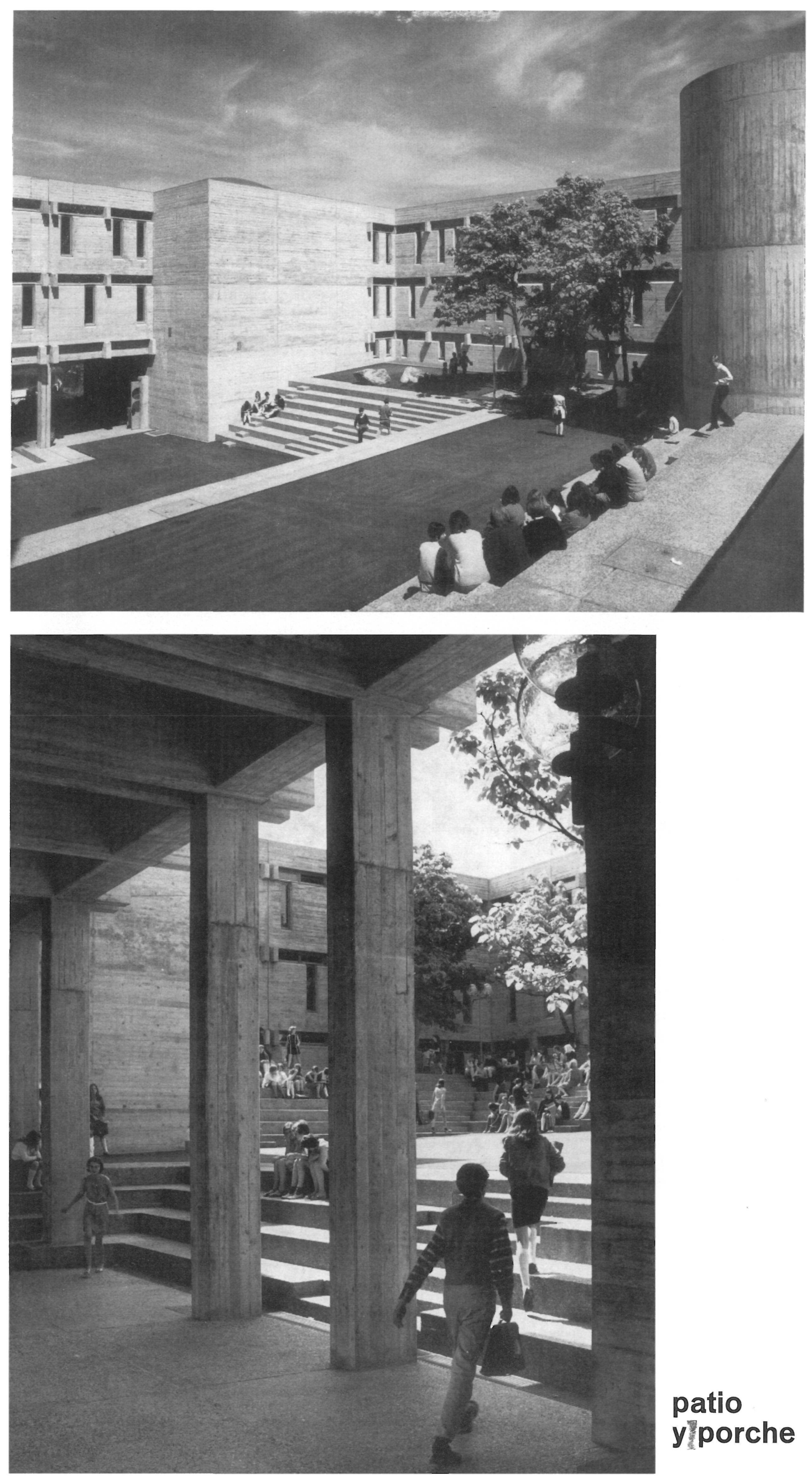

patio y porche 
emplazamiento
1. Calle Dübendorf.-2. Parada de autobús. - 3. Pavimento para coches colegio - - Patio Pabellones de gimnasia. -6. Colegio.-7. Jardín de Calle Stettbach.

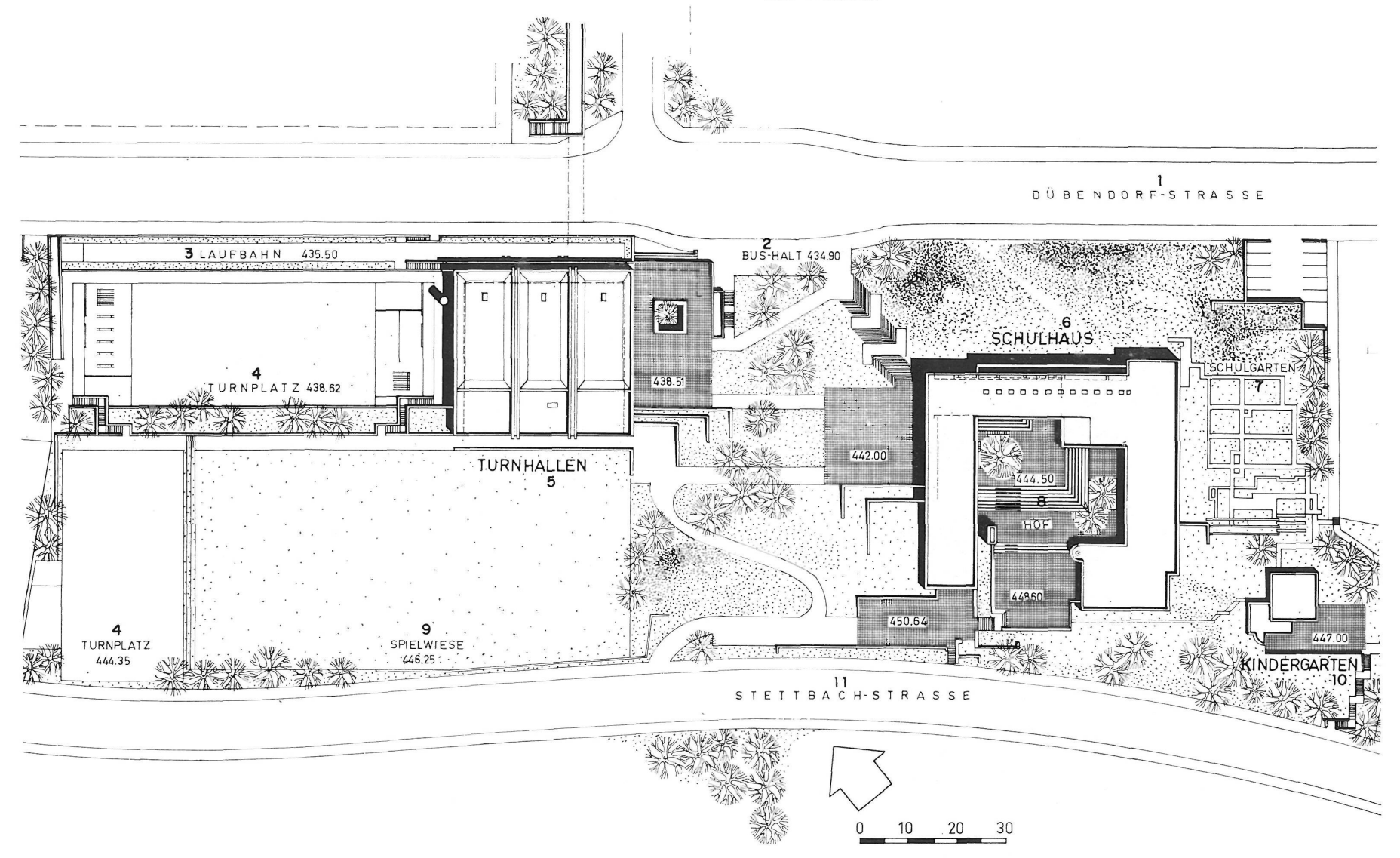

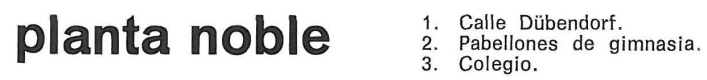

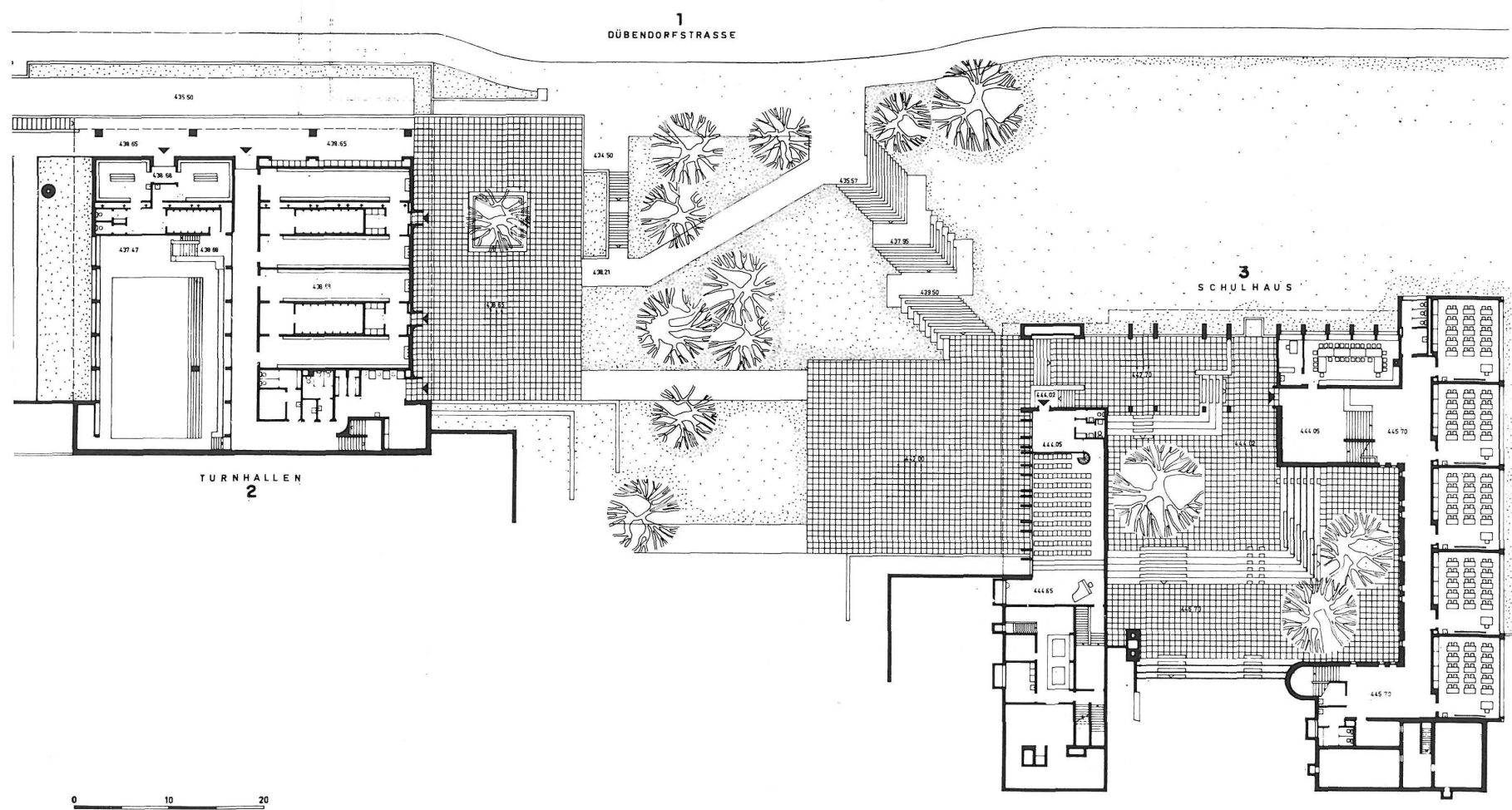




\section{planta primera}

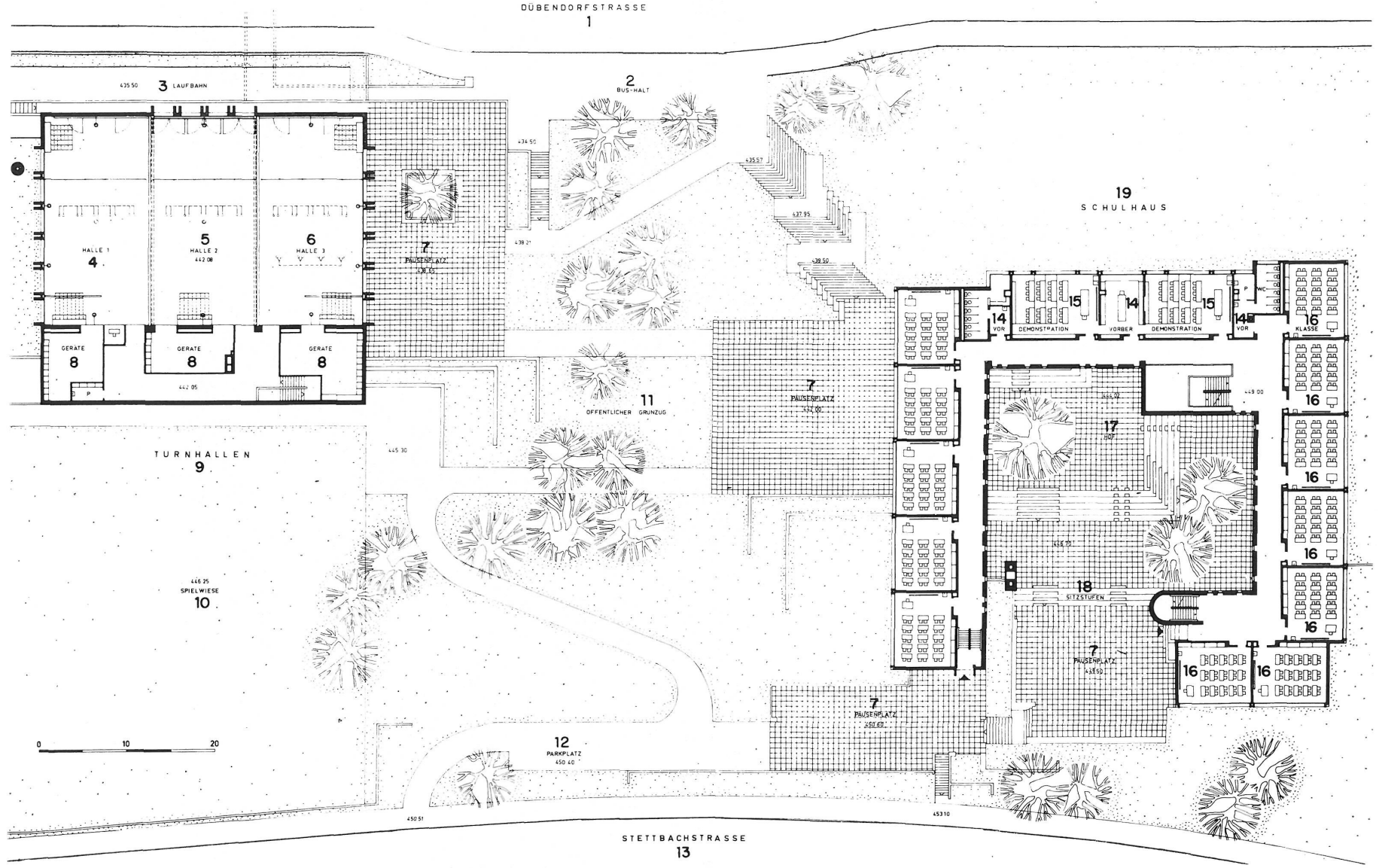

1. Calle Dübendorf.-2. Parada de autobús.-3. Pavimento para coches.-4. Pabellón 1.-5. Pabellón 2.-6. Pabellón 3.-7. Recreo.- 8. Aparatos.-9. Pabellón de gimnasia.-10. Campo de juego.-11. Zona verde.-12. Aparasientos.-19. Colegio.
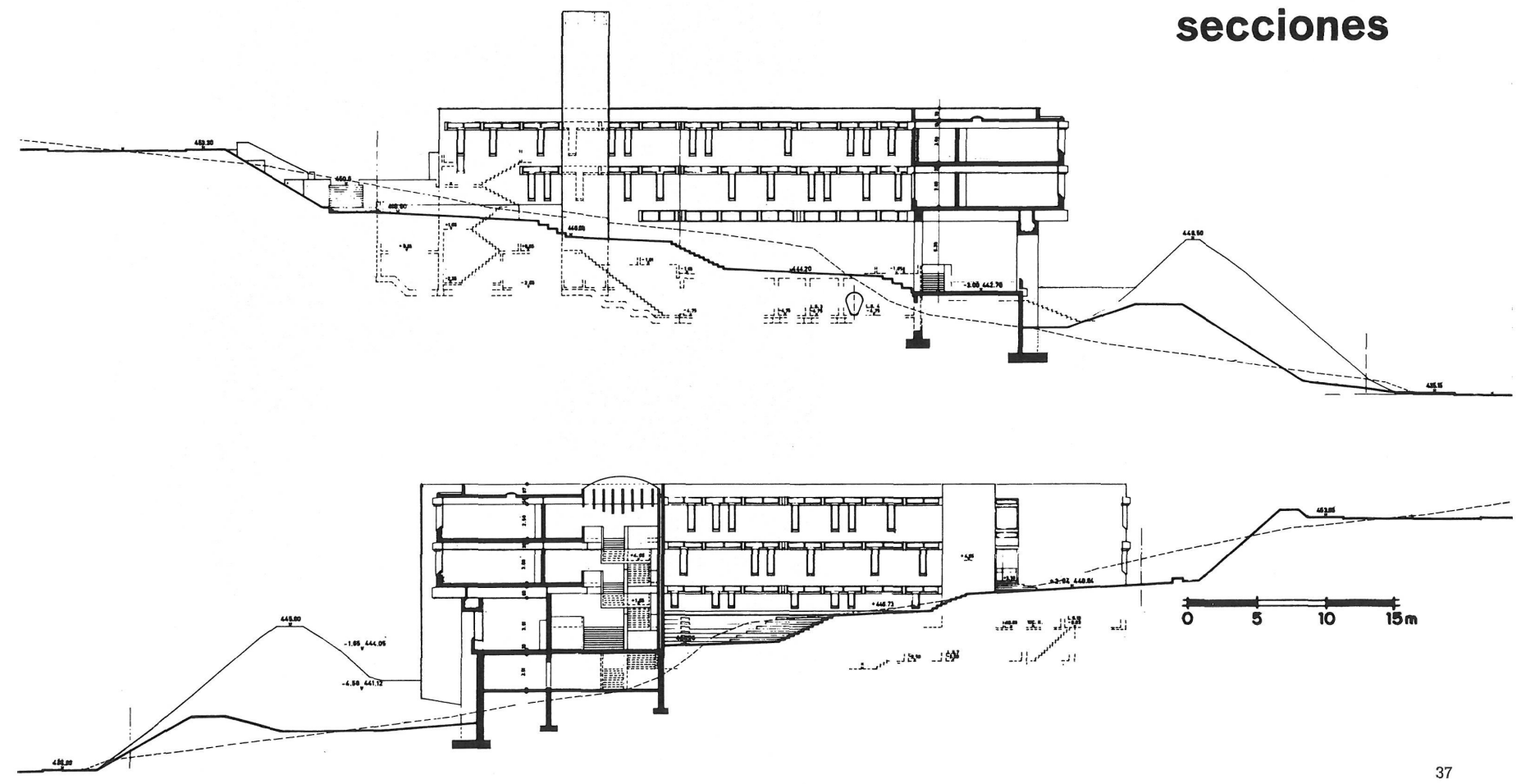


\section{sección-perspectiva}

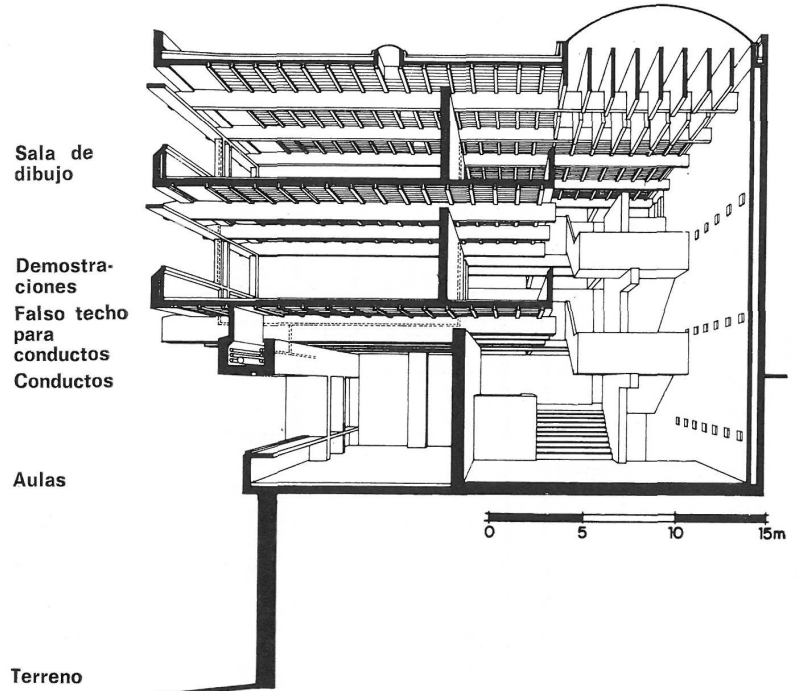

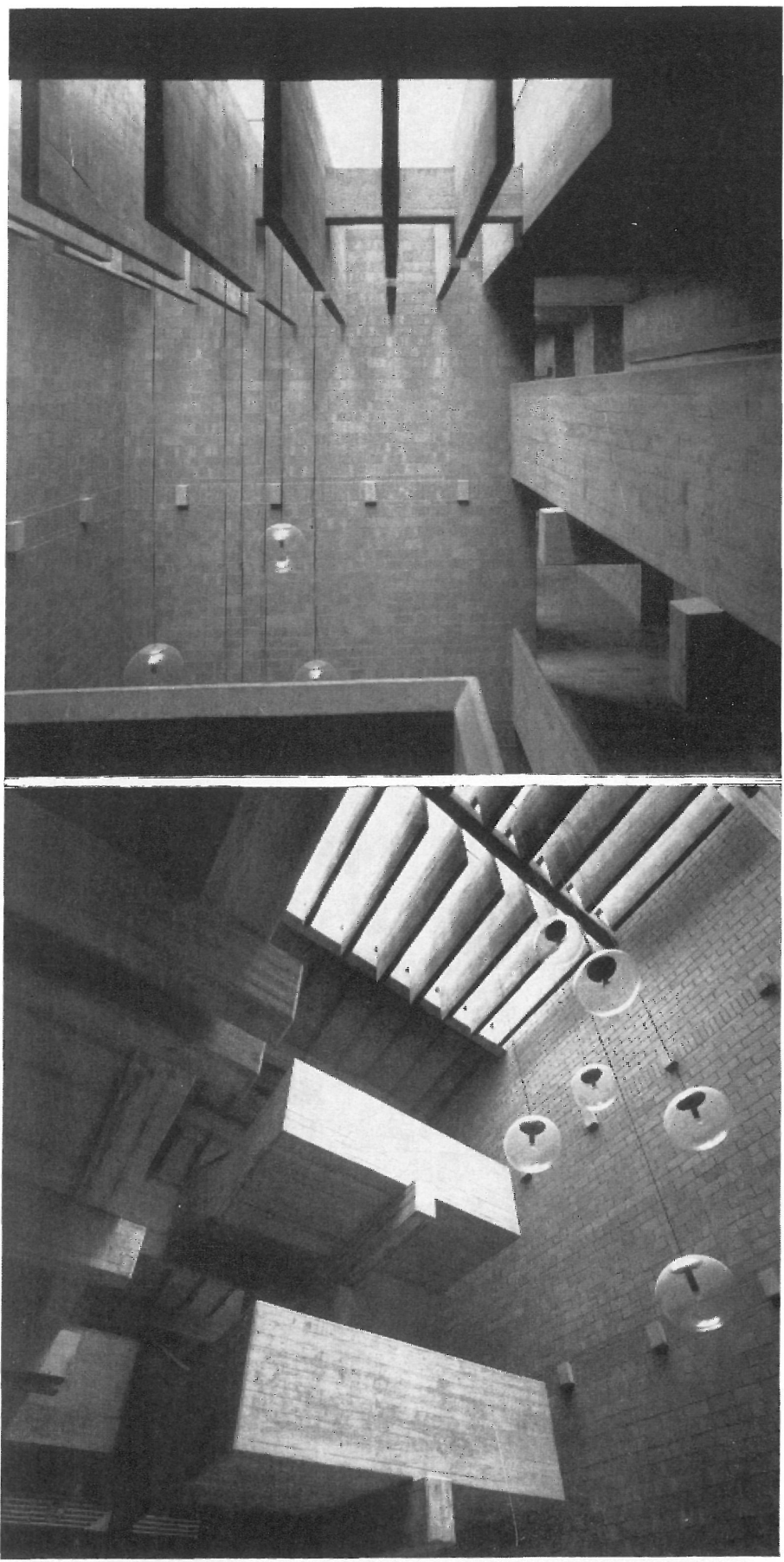

Los niveles de la construcción se adaptan y sacan partido de la fuerte pendiente del terreno, permitiendo accesos exteriores a distintas zonas del edificio desde diversos niveles.

En conjunto, el programa al que se ajusta el proyecto afecta a tres objetivos de distinta función, conformados según cuerpos de edificación independientes:

En primer lugar, la escuela secundaria propiamente dicha, con capacidad para 720 alumnos, constituye el núcleo central del proyecto. El edificio se ha resuelto en tres niveles que adoptan en planta la forma de una "U», en cuyo centro queda definido un amplio patio ajardinado, destinado al recreo de los alumnos y en el que se destaca un aprovechamiento estético de los desniveles. En el interior alberga 24 aulas para clases teóricas, 4 salas para labores manuales feme-

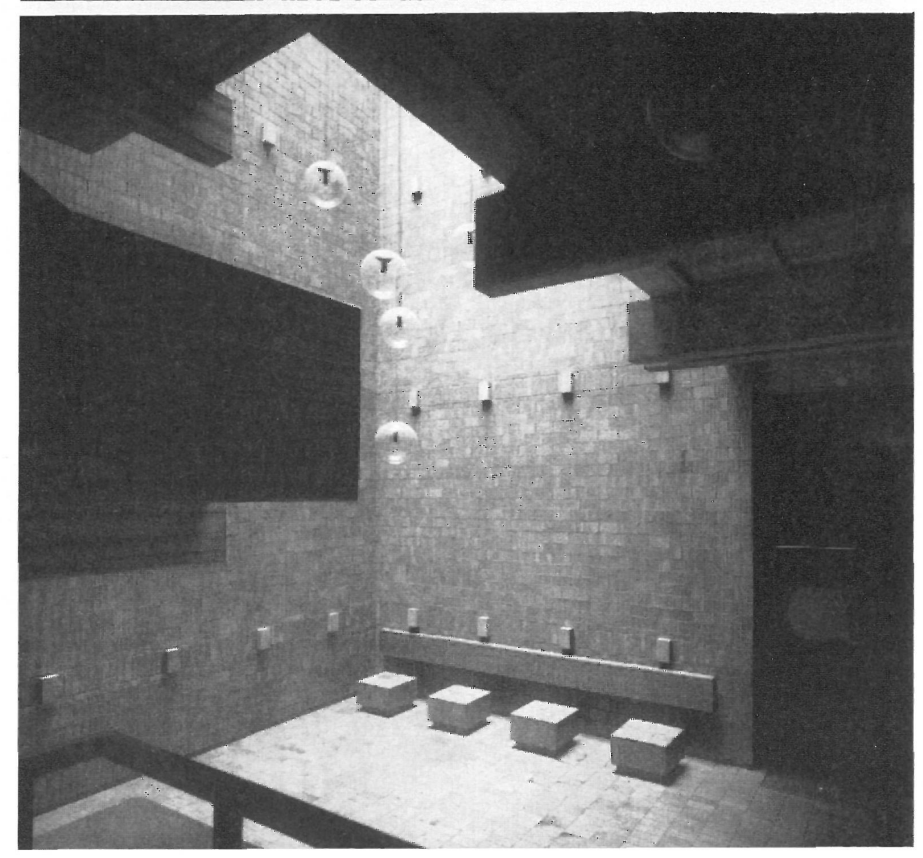




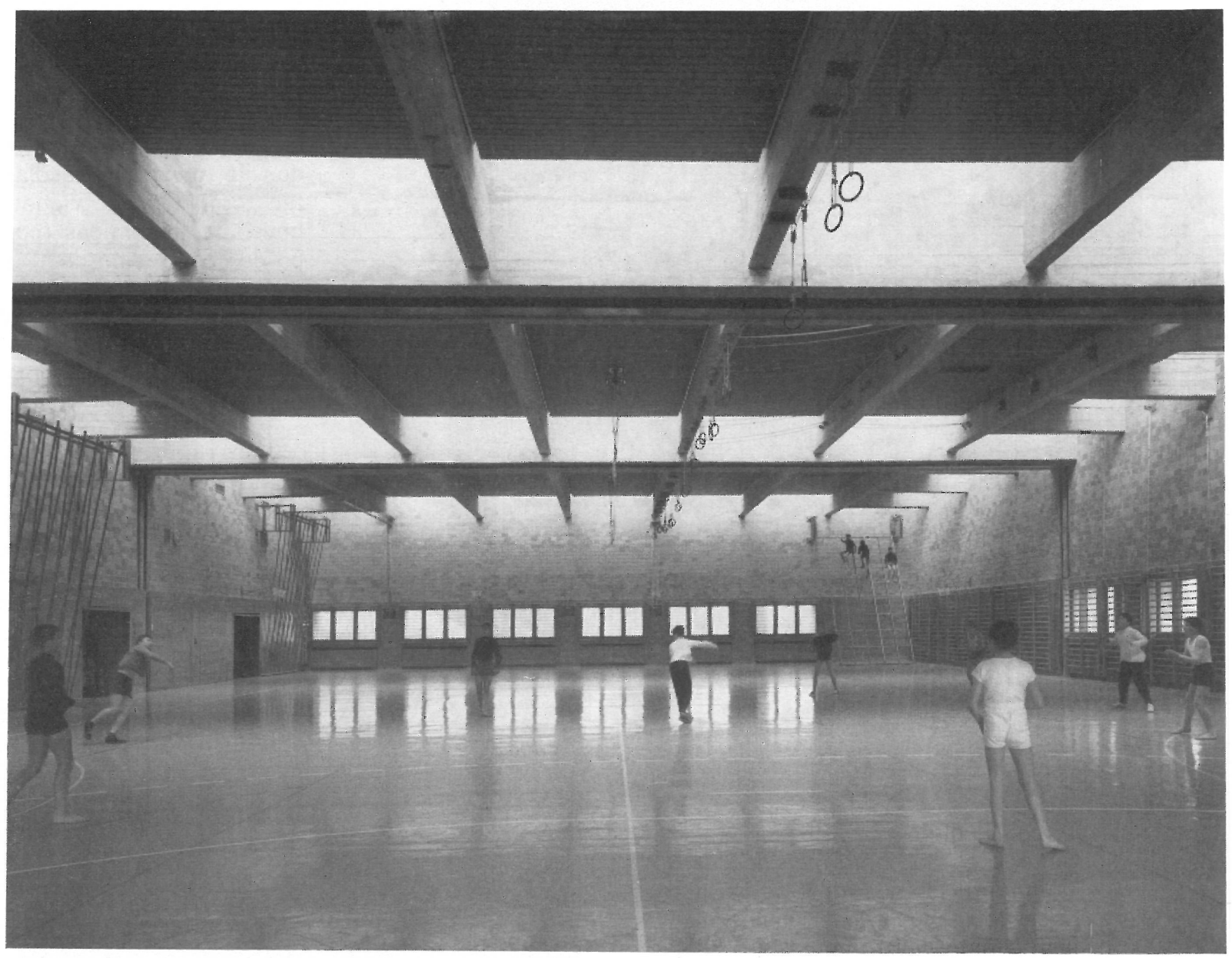

ninas, un salón para clases de canto con escenario, locales para dibujo y enseñanzas prácticas, y un taller de carpintería con depósito de materiales anexo. Para el personal docente cuenta, asimismo, con una sala de profesores y un despacho de dirección. La zona de servicios incluye una portería, diversos cuartos de instalaciones y refugios antiaéreos.

El complejo del gimnasio, separado del grupo escolar por un espacio verde público, conforma el segundo núcleo en importancia del proyecto. La construcción de dos plantas aloja tres salas de gimnasia, equipadas con tabiques divisorios que pueden elevarse, por medios electromecánicos, para dar lugar a un único espacio (de $36 \times 24 \mathrm{~m}$ ) utilizable en competiciones. Junto a estas salas se encuentran 4 vestuarios accesibles directamente desde el exterior, así como duchas y aseos para los alumnos. Los profesores disponen de servicios independientes: una piscina (de $16 \times 8 \mathrm{~m}$ ) con duchas, aseos y vestuarios. Incorporados en el mismo recinto del gimnasio se han emplazado: una estación sanitaria de primeros auxilios (con capacidad para 130 camas), una biblioteca, dos talleres para trabajos en metal, una nave para el aparcamiento (de 130 bicicletas), y locales para la delegación de obras públicas y los servicios de jardinería y mantenimiento de los campos de juego. 


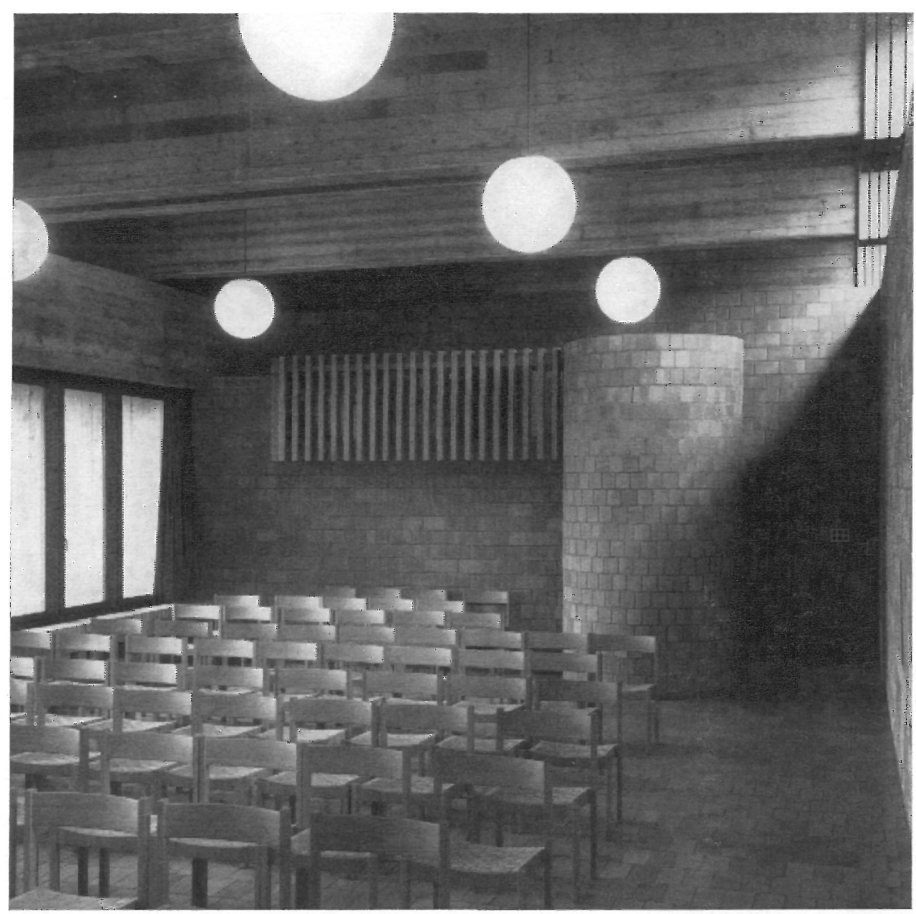

fin de obtener un efectivo aislamiento acústico. Los forjados del complejo escolar están formados por viguetas y bovedillas cerámicas (con capa de compresión de $12 \mathrm{~cm}$ ), apoyadas sobre vigas de hormigón (de $50 \mathrm{~cm}$ de canto); mientras que el del gimnasio es una losa de hormigón con placas de Durisol (de $3,5 \times$ $\times 0,5 \times 0,12 \mathrm{~m}$ ) sobre las jácenas. Las cubiertas son de material plástico con recubrimiento de grava en el edificio escolar y en el gimnasio, y con protecciones de hormigón contra las filtraciones en la guardería infantil.

El tercer núcleo, más pequeño que los anteriores, lo conforma una guardería infantil dotada de vestuarios, equipos de juegos y de trabajos manuales - tanto dentro como fuera del recinto-, y cuartos de aseo.

En el espacio exterior que circunda las construcciones se han instalado campos de recreo y paseos (con una superficie próxima a los $3.150 \mathrm{~m}^{2}$ ), una zona drenada (de $25 \times 46 \mathrm{~m}$ ), un campo para gimnasia (de $25 \times 4,7$ metros), utilizable en invierno como pista de patinaje sobre hielo, una pista para carreras (de $100 \mathrm{~m}$ ), aparatos de gimnasia, instalaciones para saltos y lanzamientos, y una pradera (de $4.000 \mathrm{~m}^{2}$ J para juegos al aire libre.

El material predominante en la construcción, tanto en el aspecto estructural como en el tratamiento de fachadas y acabados interiores, es el hormigón armado. Así, los cerramientos perimetrales se constituyeron con un muro de hormigón (de $20 \mathrm{~cm}$ de espesor), una capa aislante (de 3 centímetros) y un tabicón (de medio pie). En la distribución interior se emplearon bloques estriados de hormigón (de un pie de espesor), con el

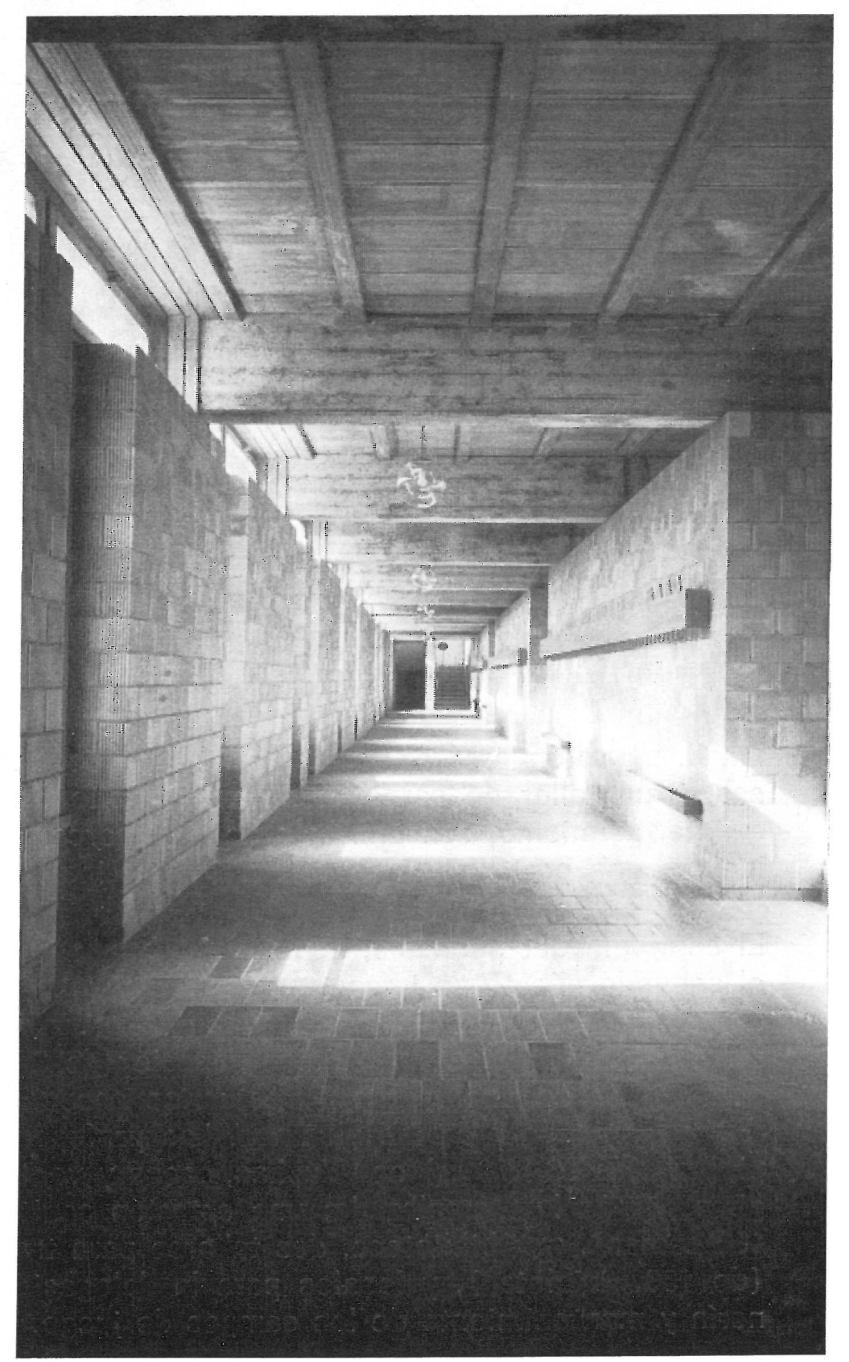

FOTOS: F. MAURER SWB 
La edificación se destaca por unos acabados sobrios y equilibrados que, tanto en el interior como en el exterior provienen, básicamente, de la exposición de los propios elementos estructurales. De este modo, las vigas de hormigón de $50 \mathrm{~cm}$ de canto, dispuestas en luces de $3 \mathrm{~m}$, permanecen vistas, quedando igualmente patentizadas las viguetas y bovedillas del forjado cerámico de los techos. En el tratamiento del hormigón, en pilares, muros, vigas y escaleras, se advierte un buen aprovechamiento de la riqueza plástica del material, dando origen a un interesante movimiento de volúmenes en el interior, así como en las fachadas. El acabado interior de los muros perimetrales se resuelve mediante un friso de madera, con capa de lana de vidrio como aislante térmico y acústico. Los tabiques interiores se forman, en la mayoría de los casos, a base de los bloques estriados vistos, excepto en las zonas de aulas, donde se revisten con un enlucido de yeso. Los pavimentos, distintos de acuerdo con su función, son de: linóleo en los salones de clase, de plástico fundido sin juntas en las cocinas y aseos, y de PVC sobre base elástica en la zona de gimnasio. Para la carpintería se han utilizado maderas de roble y de abeto, tratadas en su color, en puertas y ventanas, respectivamente. En el gimnasio, los muros perimetrales van revestidos, hasta $2,50 \mathrm{~m}$ de altura, con paneles de roble, mientras que el techo se ha acondicionado con paneles acústicos de madera aglomerada.

La iluminación artificial se consigue con lámparas esféricas, suspendidas, de cristal opalino, en las salas de clase, y transparentes, en los vestíbulos y pasillos. Tanto en el gimnasio como en los espacios de circulación de la escuela - escaleras, vestíbulos, pasillos, etc.-_, la iluminación natural se obtiene con lucernarios cenitales. En el caso de la escalera, la luz natural, que proviene de una bóveda de metacrilato realizada en la cubierta, es difuminada por una serie de lamas de hormigón, lográndose un buen acabado de formas que se integran a los volúmenes de hormigón de la propia escalera.

En cuanto a instalaciones, el edificio escolar y el gimnasio cuentan con dos calderas de carbón para la calefacción y otra alimentada por fuel-oil para la producción de agua caliente. Del mismo modo, la piscina de profesores y las duchas se abastecen de agua caliente procedente de una caldera combinada de fuel-oil.

Todos los locales están adecuadamente ventilados, bien con sistemas naturales o a base de extractores mecánicos, según las necesidades de renovación ambiental de cada zona, que llega en algunos casos hasta nueve veces por hora.

\section{résumé}

\section{Ecole secondaire à Stettbach.} Zurich Suisse

Rudolf et Esther Guyer, architectes

Cet ensemble scolaire se compose de trois noyaux nettement différenciés et indépendants, séparés entre eux par des espaces
verts, publics ou privés. Les bâtiments oc verts, publics ou privas. Les batiments oc cupent $10 \%$ du terrain, le restins et terrains de jeux.

Le noyau principal de l'ensemble est l'école secondaire pour 720 élèves, constitué par fonctions complémentaires, telles que des ourrages de dame, dessin, enseignements pratiques, menuiserie, magasins et locaux pour professeurs, direction et services.

Le gymnase comprend trois salles pour des exercices gymnastiques, qui peuvent se sports de compétition, une infirmerie pour les premiers soins, une bibliothèque, des ateliers, un parking pour bicyclèttes et des services annexes. Le noyau destiné au jardin d'enfants se
compose d'un équipement de jeux, vestiaires et toilettes.

Le bâtiment se distingue à l'extérieur pour l'équilibre assuré entre las bâtiments exépaces verts et les cours en plein air. l'intérieur révèle une utilisation maximal de la richesse plastique des propres éléments structuraux -béton aux murs, poteaux et poutres, planchers céramiques. etc.-, qui restent apparents, donnant

\section{summary}

Secondary school in Stettbach. Zurich - Switzerland

Rudolf and Esther Guyer, architects

This school complex consists of three clearly defined and independent buildings, separated by public or private gardens. The buildings occupy $10 \%$ of the lot, with the rest of the land
and play areas.

The main building of the school complex is the secondary level school, with capacity for 720 students, 24 classrooms and various rooms for complementary functions such as domestic science, art, work rooms, carpentry, storerooms, and areas for teachers, administration offices and services.

The gymnasium has three gymnastic exercise halls, which can be converted into on aid station, a library, workshops, bicycle parking area and complementary services.

The building to be used as a kindergarden has playrooms, dressing rooms and bath

The exterior of the building is outstanding as a result of the balance achieved garden areas and outdoor patios. In the interior, the plastic richness of the structural elements themselves has been used to the maximum advantage - concrete fo the walls, columns and beams; cerami interesting contrich

\section{zusammenfassung}

Mittelschule in Stettbach - Zuerich. Schweiz

Rudolf und Esther Guyer, Architekten

Dieser Schulkomplex besteht aus grundsătzlich unterschiedlichen und von einande gen voneinander getrennt werden, die öffentlich oder privat sind. Die Konstruktionen belegen $10 \%$ des Geländer, wobei der Rest ür Gärten und Spielplätze bestimmt ist.

Die Hauptgruppe des Schulkomplexes ist die Mittelschule, mit einem Fassungsvermögen für 720 Schüler, 24 Klassen und verschiedene Sälen für zusätzliche Tätigkeiten wie zun Beispiel fur Handarbeiten, Zeichnung, praktischer Unterricht, Tischlerei, Lage tion und Dienstpersonal.

Die Turnhalle besteht aus drei Sälen für Gimnastik, die man in eine einzige zwecks einer Sanitärer Station für die erste Hilfe, einer Bibliothek, Werkstätten, Parkplatz fü Fahrräder und annexe Dienste.

Die Gruppe, die für den Kindergarten bestimmt ist, besteht aus Spielgeräten, Umkleideräume und Waschräume.

Das Gebäude hebt sich äusserlich durch den gelungenen Ausgleich zwischen de weiten Grünanlagen und Höfen im Freien. hervor. Im Inneren bemerkt man eine maximale Ausnutzung des plastischen Reich tums der Strukturelemente -Beton be Wanden, Pfeiler und Trager; Keramikgebă. ke; usw-, die so ausgeführt sind, dass ein
interessanter formeller Entwurf entsteht. 